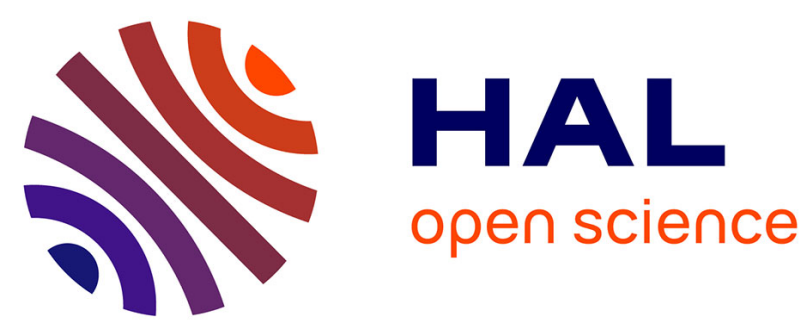

\title{
Revealing the Impact of Electrolyte Composition for Co-Based Water Oxidation Catalysts by the Study of Reaction Kinetics Parameters
}

\author{
Yan Duan, Nicolas Dubouis, Jiaqiang Huang, Daniel Alves, Dalla Corte, \\ Vanessa Pimenta, Zhichuan J Xu, Daniel Alves Dalla Corte, Alexis Grimaud
}

\section{To cite this version:}

Yan Duan, Nicolas Dubouis, Jiaqiang Huang, Daniel Alves, Dalla Corte, et al.. Revealing the Impact of Electrolyte Composition for Co-Based Water Oxidation Catalysts by the Study of Reaction Kinetics Parameters. ACS Catalysis, 2020, 10 (7), pp.4160-4170. 10.1021/acscatal.0c00490 . hal-03000051

\section{HAL Id: hal-03000051 https://hal.science/hal-03000051}

Submitted on 11 Nov 2020

HAL is a multi-disciplinary open access archive for the deposit and dissemination of scientific research documents, whether they are published or not. The documents may come from teaching and research institutions in France or abroad, or from public or private research centers.
L'archive ouverte pluridisciplinaire $\mathbf{H A L}$, est destinée au dépôt et à la diffusion de documents scientifiques de niveau recherche, publiés ou non, émanant des établissements d'enseignement et de recherche français ou étrangers, des laboratoires publics ou privés. 


\section{Revealing the impact of electrolyte composition for}

\section{Co-based water oxidation catalysts by the study of}

\section{reaction kinetics parameters}

Yan Duan ${ }^{1,2,3}$, Nicolas Dubouis ${ }^{1,4}$, Jiaqiang Huang ${ }^{1,4}$, Daniel Alves Dalla Corte ${ }^{1,4}$, Vanessa Pimenta $^{5}$, Zhichuan J. Xu ${ }^{2,3}$, Alexis Grimaud ${ }^{1,4^{*}}$

Corresponding authors: alexis.grimaud@college-de-france.fr

\section{AUTHORS ADDRESS :}

${ }^{1}$ Chimie du Solide et de l'Energie, Collège de France, UMR 8260, 75231 Paris Cedex 05,

France

${ }^{2}$ School of Materials Science and Engineering, Nanyang Technological University, Singapore 639798, Singapore

${ }^{3}$ Energy Research Institute @ NTU, ERI@N, Interdisciplinary Graduate School, Nanyang Technological University, Singapore 639798, Singapore

${ }^{4}$ Réseau sur le Stockage Electrochimique de 1‘Energie (RS2E), CNRS FR3459, 33 rue Saint Leu, 80039 Amiens Cedex, France

${ }^{5}$ Institut des Matériaux Poreux de Paris, UMR 8004 CNRS, Ecole Normale Supérieure, Ecole Supérieure de Chimie et de Physique Industrielle de Paris, PSL University, 75005, Paris, France 


\begin{abstract}
Recent studies have revealed the critical role played by the electrolyte composition on the oxygen evolution reaction (OER) kinetics on the surface of highly active catalysts. While numerous works were devoted to understand the effect of the electrolyte composition on the physical properties of catalysts' surface, very little is known yet about its exact impact on the OER kinetics parameters. In this work, we reveal that the origin for the electrolyte-dependent OER activity for Co-based catalysts originates from two different effects. Increasing the alkaline electrolyte concentration for $\mathrm{La}_{1-\mathrm{x}} \mathrm{Sr}_{\mathrm{x}} \mathrm{CoO}_{3-\delta}$ perovskites with $\mathrm{x}>0$ and for amorphous $\mathrm{CoOOH}$ increases the pre-exponential factor, which can be explained either by an increase of the concentration of active sites or by a change in the entropy of activation. However, changing the alkali cation results in a decrease of the apparent activation enthalpy for Fe-containing amorphous films, traducing a change in intermediates binding energies.
\end{abstract}

KEYWORDS: Co-based electrocatalyst, kinetic parameter, activation energy, pre-exponential factor, alkaline electrolyte, alkali-cation species 


\section{INTRODUCTION}

Water electrolysis has long been envisioned as critical for the penetration of renewable energies in the energy mix and into the chemical industry by storing electricity in the form of a chemical fuel: hydrogen. Nevertheless, water electrolysis suffers from the slow kinetics associated with its two half reactions, namely the hydrogen evolution reaction (HER) and the oxygen evolution reaction (OER). This is especially true for the OER which shows slower kinetics when compared to the HER, as well as drastic instabilities associated with the harsh oxidative and corrosive conditions employed. ${ }^{1-4}$ While early reports in the design of new OER catalysts were often focusing on the tuning of the physical properties of the electrocatalysts surface in order to improve the OER kinetics, ${ }^{1,2,56}$ recent findings pointed towards the OER being highly dependent on the electrolyte composition for a wide variety of catalysts, including Co- and Ni-based compounds. ${ }^{6-13}$ Indeed, for crystalline Co-based catalysts such as the perovskites $\mathrm{La}_{1-\mathrm{x}} \mathrm{Sr}_{\mathrm{x}} \mathrm{CoO}_{3-\mathrm{d}}$ (with $\mathrm{x} \geq 0.2$ ), ${ }^{6,14,15}$ and $\mathrm{LaNiO}_{3}{ }^{11}$ the Ruddlesden-Popper phase $\mathrm{La}_{2} \mathrm{Li}_{0.5} \mathrm{Ni}_{0.5} \mathrm{O}_{4}{ }^{11}$ or spinel based catalysts $\left(\mathrm{ZnCo}_{1.2} \mathrm{Ni}_{0.8} \mathrm{O}_{4}{ }^{7}, \mathrm{Zn}_{0.2} \mathrm{Co}_{0.8} \mathrm{OOH}^{8}, \mathrm{ZnFe}_{0.4} \mathrm{Co}_{1.6} \mathrm{O}_{4}{ }^{9}\right.$ and $\left.\mathrm{CoFe}_{0.25} \mathrm{Al}_{1.75} \mathrm{O}_{4}{ }^{10}\right)$, the OER kinetics was found to be dependent on the concentration of alkaline solution $\mathrm{MOH}$. Nevertheless, confronting this concentration dependence with results from an online electrochemical mass spectrometry (OEMS) analysis using isotopic labelling, it could be observed for the $\mathrm{La}_{1-\mathrm{x}} \mathrm{Sr}_{\mathrm{x}} \mathrm{CoO}_{3-\mathrm{d}}$ series that it is correlated with the evolution of oxygen originating from the transition metal oxide lattice. ${ }^{15}$ The conclusion was thus made that triggering the formation of electrophilic oxygen species " $\mathrm{O}^{\mathrm{n}-\sigma}$ by decreasing the Fermi level energy into oxygen bands with increasing $\mathrm{Sr}^{2+}$-substitution level would induce a change in mechanism with the proton transfer being determining. Eventually, this limitation thus gives rise to the OER to be dependent on the electrolyte concentration. Hence, for $\mathrm{LaCoO}_{3}$ the OER 
activity was found independent on the $\mathrm{KOH}$ concentration, unlike for $\mathrm{x} \geq 0.2$. This proton transfer limitation could later be alleviated by the surface decoration of $\mathrm{La}_{0.5} \mathrm{Sr}_{0.5} \mathrm{CoO}_{3-\delta}$ by phosphates moieties serving as proton relay, therefore increasing the OER kinetics ${ }^{16}$.

Moreover, the OER dependence on the electrolyte composition is not limited to crystalline catalysts and was observed for amorphous electrodeposited oxyhydroxides catalysts such as $\mathrm{CoOOH}$ which have long been described as being greatly affected by the presence of impurities in the alkaline electrolyte, especially iron impurities ${ }^{17-20}$. For this class of amorphous catalysts, not only the alkaline electrolyte concentration controls the OER kinetics, but also changing the alkali cation from $\mathrm{Li}^{+}$to $\mathrm{Cs}^{+}$was found to affect the electrocatalytic properties of such films. Furthermore, as experimentally observed by different groups, the impact of the alkali cation is more predominant for Fe-containing amorphous films and is thus dependent on the catalysts composition, alike for crystalline compounds. ${ }^{12,21,22,23,24}$ The reason for this change resides in both the change in the structure of the oxyhydroxides with a greater interlayer distance ${ }^{12,21}$ which favors the insertion of large alkali cation inside the catalyst, ${ }^{22,23}$ as well as the formation of different oxygen active species following the substitution of $\mathrm{Ni}$ or $\mathrm{Co}$ by $\mathrm{Fe}$, as previously discussed for crystalline catalysts. ${ }^{23-25}$

Hence, assessing and controlling the impact of the electrolyte composition on the OER kinetics was recently at the heart of the development of better OER electrocatalysts. Toward gaining deeper understanding of these complex effects, several operando analytical techniques as well as computation works were carried out to study the impact of the cations and/or electrolyte concentration on the physical properties of catalysts and/or the modification of surface intermediates. ${ }^{1925-30}$ However, very little is known yet about the exact correlation existing between these interfacial properties and the OER kinetics parameters, hampering further 
improvement of efficient electrocatalysts. Hence, in order to decipher the origin for the modification of the OER activity as a function of the electrolyte nature, we embarked into a systematic temperature dependence study.

For a heterogeneous electrocatalytic reaction, the catalytic current is given by the product of the rate of the reaction and the concentration of active sites:

$$
j=n F \theta_{O} a_{R} * k
$$

with $j$ the current density, $n$ the number of electrons exchanged during the reaction, $F$ the Faraday constant, $\theta_{O}$ the concentration of active sites at the electrode surface, $a_{\mathrm{R}}$ the activity of the reactant in solution and $k$ the rate constant for the reaction. In many heterogeneous electrocatalytic reactions, one electrochemical step involving a single soluble species and a single type of active site is found to be the rate determining step (rds) dictating the overall reaction kinetics, ${ }^{31,32}$ assumption which is considered to hold for the OER for which surface deprotonation $\left(* \mathrm{OH}=* \mathrm{O}+\mathrm{H}^{+}+\mathrm{e}^{-}\right)$and $\mathrm{O}-\mathrm{O}$ bond formation $\left(* \mathrm{O}+\mathrm{OH}^{-}=* \mathrm{OOH}+\mathrm{e}^{-}\right)$are often found as rds. ${ }^{14}$ Following the transition state theory, the rate constant of the reaction is given by the following equation:

$$
k=\kappa \frac{k_{B} T}{h} \exp \left(\frac{-\Delta G^{*}}{R T}\right)
$$

with $\kappa$ the transmission coefficient, $k_{B}$ the Boltzmann's constant, T absolute temperature, $h$ the Plank constant and $\Delta G^{*}$ the activation Gibb's free energy. As for heterogeneous electrocatalytic reactions the reactant and the electrode are close to each other, the transmission coefficient is often considered to be unity, ${ }^{33}$ thus no significant variation of the latter one with the temperature or the electrode composition is expected. 
However, while the rate constant is given by the product of the activation Gibb's free energy of the reaction and the transmission coefficient for electron transfer following equation (2), experimentally assessing the activation Gibb's free energy is rather challenging ${ }^{2}$. Instead, reaction rates are often compared by assessing the activation energy $E_{a}$ as given by the Arrhenius equation:

$$
E_{a}=R T^{2}\left(\frac{d \ln k}{d T}\right)
$$

Leading, after integration, to:

$$
k=A \exp \left(\frac{-E_{a}}{R T}\right)
$$

with $E_{a}$ the activation energy, $\mathrm{R}$ the gas constant and $\mathrm{A}$ the pre-exponential factor. Thus, identifying the experimental Arrhenius equation with the transition state theory leads to:

$$
A=\kappa \frac{k_{B} T}{h} e \exp \left(\frac{\Delta S^{*}}{R}\right)
$$

and

$$
E_{a}=R T+\Delta H^{*}
$$

with $\Delta S^{*}$ the entropy of activation and $\Delta H^{*}$ the enthalpy of activation (full derivation is given in the Supplementary Information). Overall, the current density as defined in equation (1) can then be expressed as follow:

$$
j=n F \theta_{O} a_{R} \kappa \frac{k_{B} T}{h} \exp \left(\frac{\Delta S^{*}}{R}\right) \exp \left(\frac{-E_{a}}{R T}\right)
$$

or

$$
j=n F A_{p p} \exp \left(\frac{-E_{a}}{R T}\right)
$$


with

$$
A_{\text {app }}=n F \theta_{O} a_{R} \kappa \frac{k_{B} T}{h} e \exp \left(\frac{\Delta S^{*}}{R}\right)
$$

$A_{a p p}$ being the "apparent" pre-exponential factor that includes all the entropic terms (the concentration terms $\theta_{O} a_{R}$ and the entropy of activation $\Delta S^{*}$ ). Furthermore, following the Bronsted-Evans-Polanyi (BEP) relation, the energy of formation of the surface intermediates involved into the OER scales linearly with the logarithm of the activation energy. For a given family of OER catalysts, if the elementary rate determining step is not modified, it is likely that the BEP relation holds. ${ }^{34}$ Therefore, when comparing the activation energies with the logarithm of the rate of the reaction, a linear trend should be obtained if the rate is only controlled by the energy of formation of the intermediates. Any deviation from this colinearity would then be explained by the modification of the $A_{a p p}$ that can origin from different factors. First, the concentration of active sites, which must be accurately determined for each catalyst prior to the reaction, can be modified under operating conditions. Second, the entropy of activation and the transmission coefficient can be dependent on the operating conditions (electrolyte, operating potential, etc.). Finally, the activity of the soluble reactive species at the interface may vary with the bulk concentration and/or the applied potential or the potential of zero charge of the catalyst.

Therefore, when screening a wide array of catalysts with fixed operating conditions, we expect to measure a linearity between the activation energy and the logarithm of the rate of the reaction (normalized current density). However, when studying the impact of the electrolyte on the rate of an electrocatalytic process, this linearity may not hold since the pre-exponential factor might be modified. In this study, we therefore compare changes in experimentally assessed activation energies and apparent pre-exponential factors for a series of Co-based catalysts in different 
alkaline electrolytes. Doing so, we aim at revealing if the modification of the OER kinetics as a function of the electrolyte originates from a change in the energy of surface intermediates, i.e. in the activation energy, or rather from a change in the apparent pre-exponential factor.

\section{EXPERIMENTAL SECTION}

Materials. Cobalt (II) nitrate hexahydrate $\left(\mathrm{Co}\left(\mathrm{NO}_{3}\right)_{2}\right.$, reagent grade, 98\%, Sigma-Aldrich), Iron (II) chloride $\left(\mathrm{FeCl}_{2}\right.$, anhydrous, 99.5\%, Alfa Aesar), Lanthanum (III) oxide $\left(\mathrm{La}_{2} \mathrm{O}_{3}\right.$, Reacton® 99.9\%, Alfa Aesar), Strontium carbonate $\left(\mathrm{SrCO}_{3}, \geqslant 99.9 \%\right.$ trace metals basis, Sigma-Aldrich), Cobalt (II, III) oxide $\left(\mathrm{Co}_{3} \mathrm{O}_{4}, 99.7 \%\right.$ metals basis, Alfa Aesar), Nickel(II) hydroxide, $\left(\mathrm{Ni}(\mathrm{OH})_{2}\right.$, Ni 61\%, Alfa Aesar), Lithium hydroxide (LiOH, anhydrous, 99.995\%, Alfa Aesar), Sodium hydroxide pellets (NaOH, Sigma-Aldrich), Potassium hydroxide (KOH, 99.99\% purity, SigmaAldrich), Cesium hydroxide hydrate $(\mathrm{CsOH}, 99.9 \%$ (metals basis) Alfa Aesar) and Tetramethylammonium hydroxide solution (TMAOH, ACS reagent Sigma-Aldrich) were purchased from commercial suppliers. Milli-Q water of $18.2 \mathrm{M} \Omega \cdot \mathrm{cm}$ was used in all experiments.

Synthesis of $\mathbf{L a}_{1-x} \mathbf{S r}_{\mathbf{x}} \mathrm{CoO}_{3}$. Perovskites $\mathrm{LaCoO}_{3}, \quad \mathrm{La}_{0.8} \mathrm{Sr}_{0.2} \mathrm{CoO}_{3}, \quad \mathrm{La}_{0.6} \mathrm{Sr}_{0.4} \mathrm{CoO}_{3}$ and $\mathrm{La}_{0.5} \mathrm{Sr}_{0.5} \mathrm{CoO}_{3}$ were synthesized by conventional solid-state route. Stoichiometric amounts of $\mathrm{La}_{2} \mathrm{O}_{3}, \mathrm{SrCO}_{3}, \mathrm{Co}_{3} \mathrm{O}_{4}$ previously dehydrated were thoroughly ground, pressed into pellets and annealed in air at $950{ }^{\circ} \mathrm{C}$ for $12 \mathrm{~h}$. $\mathrm{La}_{0.8} \mathrm{Sr}_{0.2} \mathrm{CoO}_{3}, \mathrm{La}_{0.6} \mathrm{Sr}_{0.4} \mathrm{CoO}_{3}$ and $\mathrm{La}_{0.5} \mathrm{Sr}_{0.5} \mathrm{CoO}_{3}$ were ground and annealed for a second time in air at $950{ }^{\circ} \mathrm{C}$ for another $12 \mathrm{~h}$ and slowly cooled down to room temperature.

Film deposition of $\mathrm{Co}(\mathrm{OH})_{2}$ and $\mathrm{Fe}_{0.5} \mathrm{Co}_{0.5}(\mathrm{OH})_{2}$. Prior to the film deposition, the glassy carbon insert for the rotating disk electrodes were cleaned by cycling 10 times in alkaline 
solution with a potential range of 1.1-1.7 V vs. RHE. Prior to the electrochemical deposition, the electrodes were polished and ultrasonically washed by ethanol and $18.2 \mathrm{M} \Omega \cdot \mathrm{cm}$ water. Films of $\mathrm{Co}(\mathrm{OH})_{2}$ were cathodically deposited in an argon saturated $10 \mathrm{mM} \mathrm{Co}\left(\mathrm{NO}_{3}\right)_{2}$ solution at a current density of $50 \mu \mathrm{A} \mathrm{cm}^{-2}$ for $600 \mathrm{~s}$. After deposition, films were rinsed by dipping in deionized water $(18.2 \mathrm{M} \Omega \cdot \mathrm{cm})$. Prior to the OER measurements, the as-deposited electrodes were first cycled in "Fe-free" alkaline solution at $100 \mathrm{mV} \mathrm{s}^{-1}$ with a potential range of 1.1-1.7 V vs. RHE for 20 cycles for conditioning.

Films of $\mathrm{Fe}_{0.5} \mathrm{Co}_{0.5}(\mathrm{OH})_{2}$ were cathodically deposited in an argon saturated $6 \mathrm{mM} \mathrm{Co}\left(\mathrm{NO}_{3}\right)_{2}+4$ $\mathrm{mM} \mathrm{FeCl} 2$ solution at a current density of $50 \mu \mathrm{A} \mathrm{cm}{ }^{-2}$ for $600 \mathrm{~s}$. After deposition, films were rinsed by dipping in deionized water $(18.2 \mathrm{M} \Omega \cdot \mathrm{cm})$. The as-deposited electrodes were firstly cycled in non-purified alkaline solution at $100 \mathrm{mV} \mathrm{s}^{-1}$ with a potential range of 1.1-1.7 $\mathrm{V}$ vs. RHE for 20 cycles for conditioning. While the nominal stoichiometry of $\left.\mathrm{Co}_{0.6} \mathrm{Fe}_{0.4(} \mathrm{OH}\right)_{2}$ was initially targeted owing to its enhanced OER activity as previously reported, ${ }^{17}$ analysis by scanning electron microscopy - energy dispersive X-ray spectroscopy (SEM-EDX) revealed a deviation from this nominal composition and the electrodeposition of a film with the composition $\mathrm{Fe}_{0.5} \mathrm{Co}_{0.5}(\mathrm{OH})_{2}$.

Electrochemistry measurement. Electrodes were prepared for the perovskites series by dropcasting an ink containing the oxide catalyst powder on a glassy carbon electrode. $10 \mu \mathrm{L}$ of ink with a mass ratio of 5:1:1 of oxide catalyst to acetylene black carbon to Nafion ${ }^{\circledR}$ were loaded on the polished glassy carbon electrode surface $\left(0.196 \mathrm{~cm}^{2}\right)$. Oxygen evolution reaction (OER) measurements were performed on a biologic VSP potentiostat with a rotating disk electrode setup (RDE, PINE Inc, US.). A three-electrode configuration was adopted using glassy carbon electrode as working electrode, an $\mathrm{Ag} / \mathrm{AgCl}$ saturated reference electrode and a $\mathrm{Pt}$ wire as 
counter electrode. The $\mathrm{Ag} / \mathrm{AgCl}$ reference electrode was calibrated using a reversible hydrogen electrode (RHE) (HydroFlex, Gaskatel, Germany) before and after each experiment. A jacked cell connected with a chiller was used for temperature adjustment.

For the measurements of $\mathrm{CoOOH}$ and perovskites $\mathrm{LaCoO}_{3}, \mathrm{La}_{0.8} \mathrm{Sr}_{0.2} \mathrm{CoO}_{3}, \mathrm{La}_{0.6} \mathrm{Sr}_{0.4} \mathrm{CoO}_{3}$ and $\mathrm{La}_{0.5} \mathrm{Sr}_{0.5} \mathrm{CoO}_{3}$ in alkaline solutions, all the alkaline solutions including $\mathrm{LiOH}, \mathrm{NaOH}, \mathrm{KOH}$, $\mathrm{CsOH}$, and $\mathrm{TMAOH}$ were purified by $\mathrm{Ni}(\mathrm{OH})_{2}$ to remove $\mathrm{Fe}$ impurities. Prior to the temperature dependence study, a preconditioning step was applied during 20 scans at a scan rate of $100 \mathrm{mV} / \mathrm{s}$ in the potential range 1.1-1.7 V vs RHE. For the OER measurements, a scan rate of $10 \mathrm{mV} / \mathrm{s}$ was used for cyclic voltammetry (CV) measurements and the rotation speed was set to $1600 \mathrm{rpm}$. For this study, CV measurements were used instead of galvanostatic or potentiostatic measurements. Indeed, during potentiostatic or galvanostatic holdings, oxygen bubbles are generated on the electrode which surface is eventually partially blocked with time, as observed in Figure S1. This surface blocking is relatively limited during much shorter $\mathrm{CV}$ scans performed at $10 \mathrm{mV} / \mathrm{s}$. For the temperature dependence measurements, the temperature was increased from 10 to $60{ }^{\circ} \mathrm{C}$ with an increment of $10{ }^{\circ} \mathrm{C}$ and electrochemical tests were performed afterwards at room temperature to ensure that the electrode suffered no drastic degradation or modifications.

All electrochemical potentials here are reported against the reversible hydrogen electrode (RHE). The conversion between potentials versus $\mathrm{Ag} / \mathrm{AgCl}$ and versus $\mathrm{RHE}$ was performed by the equation below:

$\mathrm{E}($ vs RHE $)=\mathrm{E}($ vs $\mathrm{Ag} / \mathrm{AgCl})+\mathrm{E}_{\mathrm{Ag} / \mathrm{AgCl}}($ vs RHE $)$ 
and $\mathrm{E}_{\mathrm{Ag} / \mathrm{AgCl}}$ was calibrated at the different temperatures in the different solutions prior to the measurements using the RHE electrode (see above). Furthermore, the reversible potentials for the OER have been corrected as a function of the temperature ${ }^{35}$.

From the CV scans obtained at the different temperatures, Tafel curves were plotted for the OER currents which were obtained from the average between the forward and the backward scans in order to remove the capacitive current contribution, before to be corrected with the ohmic losses. The ohmic drops were corrected by subtracting the ohmic voltage drop from the measured potential, using an electrolyte resistance determined by high-frequency AC impedance, where $\mathrm{iR}$-corrected potentials are denoted as $\mathrm{E}-\mathrm{iR}$ ( $\mathrm{i}$ as the current and $\mathrm{R}$ as the electrolyte resistance). Activation energy and pre-exponential factors for the perovskites and films in electrolyte with different $\mathrm{pH}$ and cations were extrapolated from the slope and the intercept of the $\ln (\mathrm{j})$ vs. 1000/T curves. Error bars represent standard deviation from at least 3 independent measurements.

Influence of the Mass-Transport. In order to assess the kinetic parameters from the measured current density, we must first make sure that all the mass-transport limitations are discarded so that the activity of the reactant at the electrode-electrolyte interface $\left(a_{R}\right)$ can be considered as constant. The effect of the mass-transport can be quantified by considering the Koutecký-Levich equation when a RDE setup is employed: ${ }^{36}$

$$
\frac{1}{j_{\text {measured }}}=\frac{1}{j_{k}}+\left(\frac{1}{0.62 n F D^{\frac{2}{3}} v^{-\frac{1}{6}} C_{\text {bulk }}}\right) \omega^{-\frac{1}{2}}
$$

where $j_{k}$ is the kinetic current, $n$ is the number of electrons transferred, $F$ the Faraday constant, $D$ the diffusion coefficient for the reactant, $v$ the scan rate in the experiment and $\omega$ the rotation 
speed of the electrode. In the absence of mass transport limitation, the second term of the Koutecký-Levich equation should be null and the measured current density is the true kinetic current density. In the case of the alkaline OER, we first demonstrated the absence of influence of the rotation speed for the OER activity measured for different electrodes/compositions (both for dropped-casted perovskites as well as for electrodeposited materials, Figure S2). These measurements undoubtedly demonstrate that there is no mass transport limitation and that the measured current density truly reflects the OER kinetics. This is to no surprise, since the soluble reactive species involved in the OER rate determining steps are probably $\mathrm{OH}^{-}$anions $\left(\mathrm{O}^{*}+\mathrm{OH}^{-}\right.$ $=\mathrm{OOH}^{*}+\mathrm{e}^{-}$) (or maybe $\mathrm{H}_{2} \mathrm{O}$ molecules) for which the activity is large in the $\mathrm{pH}$ conditions explored $(\mathrm{pH}>13)$.

Characterization. X-ray powder diffraction (XRD) measurements were performed using a BRUKER D8 Advance diffractometer with $\mathrm{Cu} K \alpha$ radiation $\left(\lambda \mathrm{K} \alpha_{1}=1.54056 \AA\right.$, $\lambda \mathrm{K} \alpha_{2}=1.54439$ $\AA$ ). Data were collected using the $\mathrm{X}^{\prime}$ Celerator detector in the $10-85^{\circ}$ window in the $2 \theta$ range.

The specific surface area for $\mathrm{LaCoO}_{3}, \mathrm{La}_{0.8} \mathrm{Sr}_{0.2} \mathrm{CoO}_{3}, \mathrm{La}_{0.6} \mathrm{Sr}_{0.4} \mathrm{CoO}_{3}, \mathrm{La}_{0.5} \mathrm{Sr}_{0.5} \mathrm{CoO}_{3}$ perovskites were determined through Brunauer, Emmet and Teller (BET) analysis. Nitrogen porosimetry measurements were performed on a Micromeritics Triflex instrument at $77 \mathrm{~K}$, after $7 \mathrm{~h}$ outgassing at $150{ }^{\circ} \mathrm{C}$.

The electrochemically active surface area (ECSA) of $\mathrm{CoOOH}$ and $\mathrm{Fe}_{0.5} \mathrm{Co}_{0.5} \mathrm{OOH}$ were quantified based on the following equation: $\mathrm{ECSA}=\mathrm{C}_{\mathrm{DL}} / \mathrm{C}_{\mathrm{S}}$ where $\mathrm{C}_{\mathrm{DL}}$ is the non-faradaic double layer capacitance, $\mathrm{C}_{S}$ is the specific capacitance and $\mathrm{i}=v \mathrm{C}_{\mathrm{DL}}$ where $\mathrm{i}$ is the current, $v$ is the $\mathrm{CV}$ scan rate. $\mathrm{C}_{\mathrm{DL}}$ is the slope of the $\mathrm{i}$ vs $v$ curve. 


\section{RESULTS AND DISCUSSION}

Toward understanding the impact of the electrolyte on the OER kinetics for different transition metal oxides, we first reinvestigated the $\mathrm{La}_{1-x} \mathrm{Sr}_{x} \mathrm{CoO}_{3-\delta}$ perovskite series previously reported by several groups to show enhanced OER kinetics with the $\mathrm{Sr}^{2+}$ content. ${ }^{6,16,37,38}$ The OER kinetics for $\mathrm{La}_{1-\mathrm{x}} \mathrm{Sr}_{\mathrm{x}} \mathrm{CoO}_{3-\mathrm{d}}$ catalysts with $\mathrm{x}=0,0.2,0.4$ and 0.5 were first investigated in $0.1 \mathrm{M} \mathrm{KOH}$ by the means of cyclic voltammetry in the 283-333 K range (Figure 1a). Surprisingly, in this temperature range, no significant modification of the Tafel slope which should be temperature dependent is observed; these slopes are measured in the range of $72-79 \mathrm{mV} \mathrm{dec}^{-1}$ (Inset in Figure 1a and Figure S3), values which deviate from the ones predicted by microkinetics analysis. ${ }^{32,39}$ Under such conditions, it becomes rather complex to correctly extrapolate the exchange current densities from the Tafel analysis. Similar observation was previously made for other complex electrocatalytic processes such as the HER on the surface of Pt-based catalysts and, in order to tackle this issue, analysis of the HER kinetics was then done in the so-called micropolarization region. ${ }^{32,39}$ However, owing to the irreversible nature of the oxygen reactions (OER/ORR), this approach cannot be used for OER electrocatalysts. Therefore, in this work, the apparent activation energies were extracted from the current density at a fixed overpotential of $\eta=400$ $\mathrm{mV}$ rather than by using the extrapolated exchange current densities (inset in Figure 1a and Figure 1b), while the apparent pre-exponential factors were extrapolated following the method described in the Supplementary Information. Nevertheless, the activation energies and the

apparent pre-exponential factors should theoretically be dependent on the overpotential. ${ }^{40}$ However, when comparing the values estimated at $\eta=400 \mathrm{mV}$ with those extrapolated using the temperature dependence of the exchange current density as extrapolated from the Tafel slopes (Figure S4), they are relatively similar. Such observation is rather surprising as, with $400 \mathrm{mV}$ 
overpotential, one would expect the activation barrier to be lowered by $400 \mathrm{meV}\left(\sim 40 \mathrm{~kJ} \mathrm{~mol}^{-1}\right)$. This emphasizes that no direct microkinetics analysis can be made for the OER in these conditions, and that both the concentration of active sites as well as change in the exposed oxide surface can occur, as recently pointed out. ${ }^{41}$ Therefore, we believe than adopting a more phenomenological approach by extracting the activation energies and the pre-exponential factors at given values for the overpotential is a more adapted methodology.
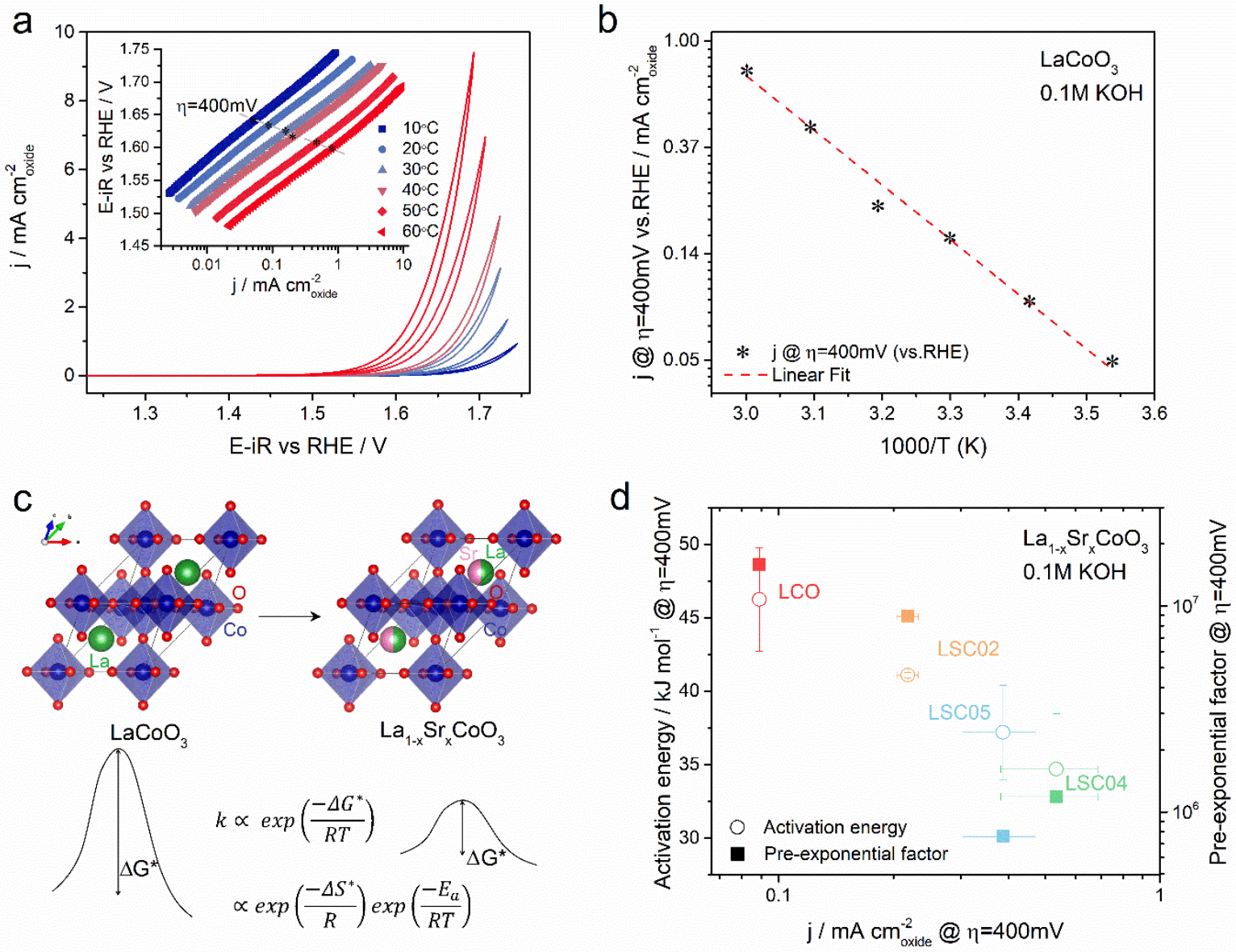

Figure 1. (a) Cyclic voltammetry (CV) measurements for $\mathrm{LaCoO}_{3}$ electrode measured at 10 $\mathrm{mV} / \mathrm{s}$ in $0.1 \mathrm{M} \mathrm{KOH}$ from 10 to $60^{\circ} \mathrm{C}$ with an increment of $10^{\circ} \mathrm{C}$. (b) Extrapolation of the apparent activation energy and the pre-exponential factor from the Arrhenius plot $\mathrm{j}$ vs 1000/T. (c) Schematic illustration for the effect of $\mathrm{Sr}^{2+}$ substitution into $\mathrm{LaCoO}_{3}$ on the activation barrier for the OER. (d) Apparent activation energies and pre-exponential factors extrapolated at $\eta=400 \mathrm{mV}$ vs. RHE for the $\mathrm{La}_{1-\mathrm{x}} \mathrm{Sr}_{\mathrm{x}} \mathrm{CoO}_{3}$ series as a function of the OER activity at $\eta=400 \mathrm{mV}$ vs. RHE measured in $0.1 \mathrm{M} \mathrm{KOH}$ at $20^{\circ} \mathrm{C}$. 
With this in mind, the OER current density for the different Co-based perovskites was then normalized by the BET surface area to estimate the rate of the reaction (equation 1) and the apparent activation energies experimentally obtained were plotted as a function of the OER current density at $\eta=400 \mathrm{mV}$ in $0.1 \mathrm{M} \mathrm{KOH}$. Doing so, it appears that when the rate of the reaction is increasing, i.e. when the normalized current density is increasing, a decrease of the experimentally determined apparent activation energies is found (Figure 1c-d). Thus, one can confirm that this methodology can capture the previously reported trend in which the partial substitution of $\mathrm{La}^{3+}$ by $\mathrm{Sr}^{2+}$ leads to an enhancement of the OER activity (note that, similarly to previous reports ${ }^{6}$, the OER activity in the $0.4 \leq \mathrm{x} \leq 0.6$ range for $\mathrm{La}_{1-\mathrm{x}} \mathrm{Sr}_{\mathrm{x}} \mathrm{CoO}_{3-\delta}$ is only marginally impacted by the substitution level). This observation is in agreement with a previous report by Bockris and Otagawa who found a linear trend between the OER activity and the activation energies measured for a wide variety of perovskites, as we recently discussed. ${ }^{42,43}$

Now turning to the evolution of the apparent pre-exponential factor as a function of the OER activity, it is found to decrease concomitantly to the decrease of the activation energies. While the decrease of the apparent pre-exponential factor should result in a decrease of the OER activity (equation 3b), the effect associated with the decrease of the activation energy is predominant on the OER kinetics for these perovskites. As previously discussed in the introduction, a modification of the apparent pre-exponential factor can result from several phenomena such as a change in the interfacial concentration of soluble species and/or active sites or a change in the entropy of activation. Here, since the electrolyte concentration is kept constant, the interfacial concentration of soluble species is not likely to drive the modifications of the apparent pre-exponential factor. Rather, the decrease of the apparent pre-exponential 
factor observed with the decrease of the activation energy measured on these catalysts may originate from a decrease of the concentration of the active sites, or of the entropy of activation for the reaction (equation $4 \mathrm{a})$.

It is noteworthy to observe that the activation energies and the pre-exponential factors evolve in a competing fashion was previously made with for instance Pt-based ORR catalysts. This effect is often referred to as a compensation effect. ${ }^{44-46}$ The physical origin for such effect is beyond the scope of this manuscript. Nevertheless, when plotting the pre-exponential factor as a function of the activation energies that were obtained for the $\mathrm{La}_{1-\mathrm{x}} \mathrm{Sr}_{\mathrm{x}} \mathrm{CoO}_{3-\delta}$ series, a linear correlation is obtained forming a so-called Meyer-Neldel plot (Figure 2). From this first study, we can however conclude, and to no surprise, that the enhancement of the OER activity within the $\mathrm{La}_{1-}$ ${ }_{x} \mathrm{Sr}_{\mathrm{x}} \mathrm{CoO}_{3-\delta}$ series arises from a modification of the enthalpy of formation of the intermediates on the surface of these perovskites which lowers the activation energy (equation $4 b$ ).

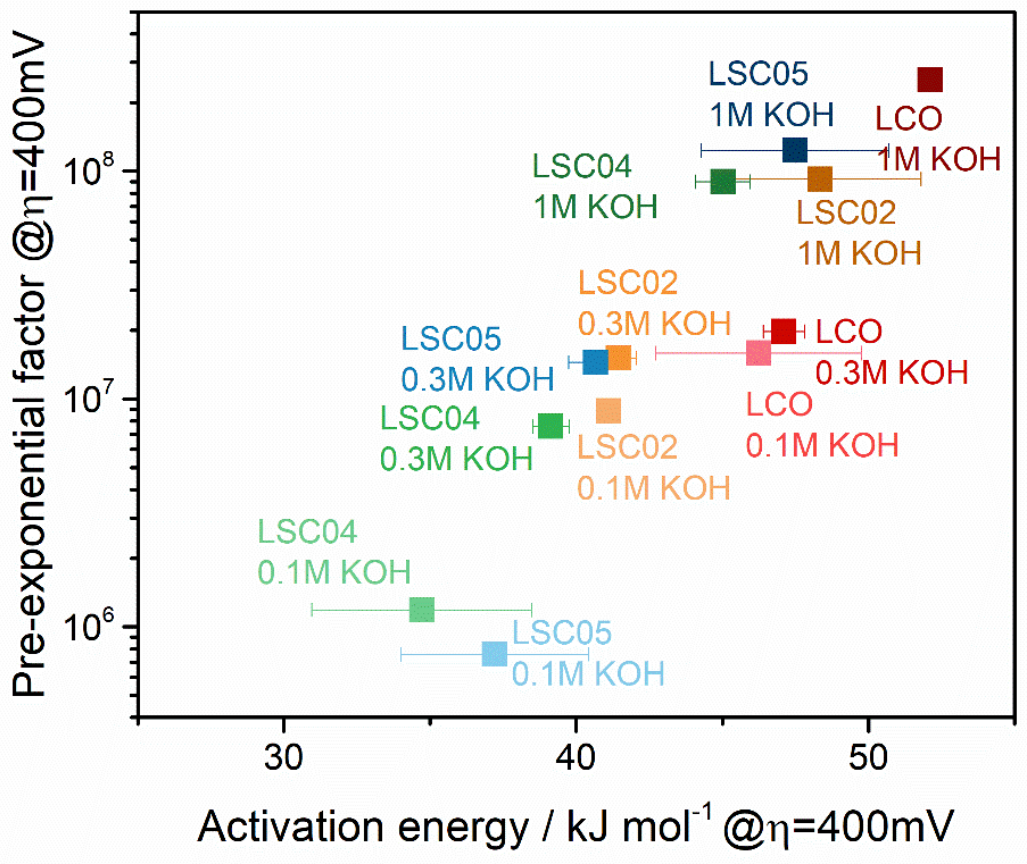


Figure 2. Pre-exponential factors plotted as a function of the apparent activation energies extrapolated at $\eta=400 \mathrm{mV}$ for the $\mathrm{La}_{1-\mathrm{x}} \mathrm{Sr}_{\mathrm{x}} \mathrm{CoO}_{3}$ series in $0.1,0.3$ and $1 \mathrm{M} \mathrm{KOH}$ from Figure $1 \mathrm{~d}$ and $3 \mathrm{a}$.

Having confirmed that modifying the electronic properties of OER catalysts directly impact the activation energy and therefore the energy of the surface intermediates, our attention then turned to the effect of the electrolyte concentration on the OER kinetics measured for Co-based catalysts. First, as previously observed, ${ }^{16,37}$ the OER activity for $\mathrm{LaCoO}_{3}$ is found independent on the concentration of $\mathrm{KOH}$ while it increases for $\mathrm{La}_{1-\mathrm{x}} \mathrm{Sr}_{\mathrm{x}} \mathrm{CoO}_{3-\delta}$ catalysts with $\mathrm{x}>0$ (Figure $3 \mathrm{a}$ ). More importantly, the enhanced OER activity for $\mathrm{La}_{1-\mathrm{x}} \mathrm{Sr}_{\mathrm{x}} \mathrm{CoO}_{3-\delta}$ catalysts with $\mathrm{x}>0$ is found to arise from an increase of the pre-exponential factor (Figure 3b). Instead, following the aforementioned compensation effect, ${ }^{46}$ the activation energy is found to increase but not sufficiently for its effect to be predominant on the OER kinetics. Similar observation was made for Pt-based oxygen reduction reaction (ORR) alloy catalysts for which the activation energy was found invariant on the composition. ${ }^{47}$ In addition to the modification of the concentration of active sites (catalyst surface deprotonation to form $* \mathrm{O}$ ) and the modification of the entropy of activation or the reaction, the increase of the electrolyte concentration may increase the concentration of soluble species at the interface. This could explain the concomitant increase of the apparent pre-exponential factor with the electrolyte $\mathrm{pH}$. However, while an increase of the apparent pre-exponential factors is measured for all the perovskites studied in this work, two different behaviors are observed for the activation energy. Hence, for the catalysts that exhibit a poor activity, the increase of the pre-exponential factor with the electrolyte $\mathrm{pH}$ is almost fully compensated by an increase of the activation energy. On the contrary, for the best catalysts, even if a slight increase of the activation energy is observed, the drastic increase of the apparent pre- 
exponential factor with the electrolyte concentration governs the performances of these catalysts. Finally, similar observation is made for other alkali-cation where, for instance, the increase of the OER activity as a function of $\mathrm{LiOH}$ concentration is explained, alike for $\mathrm{KOH}$, by an increase of the pre-exponential factor rather than by a decrease of the apparent activation energy (Figure 3b). Overall, while the concentration has a great influence on the OER kinetics, the nature of the alkali-cation has a rather limited impact on the OER kinetics for these Co-based perovskites (Figure 3c and Figure S5-6). This is especially true when compared to the effect reported for amorphous Co-based electrodeposited films ${ }^{48}$ which will be discussed below.
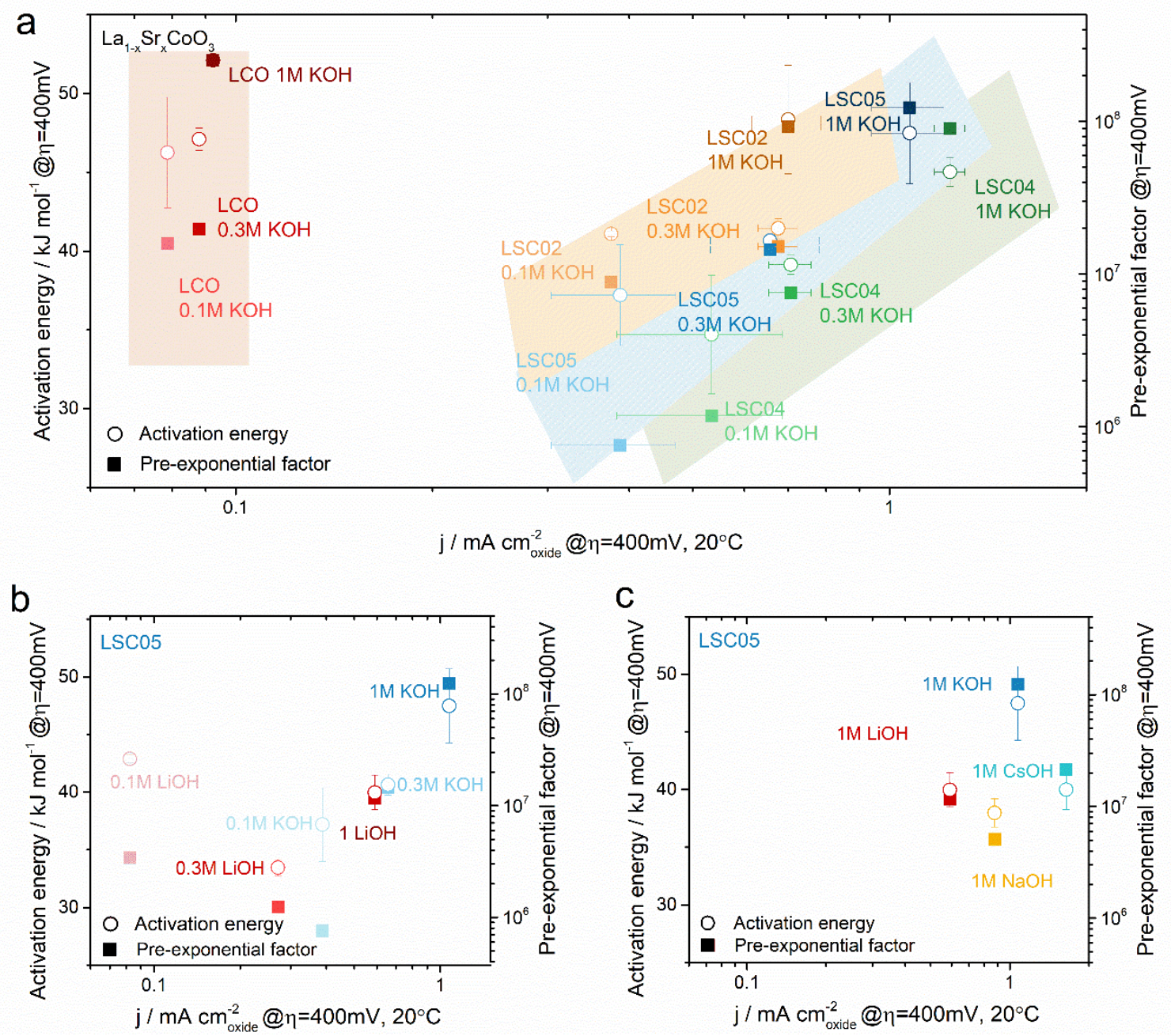
Figure 3. Apparent activation energies and pre-exponential factors as a function of the OER activity measured at $20^{\circ} \mathrm{C}$ at $\eta=400 \mathrm{mV}$ (a) in $0.1,0.3$ and $1 \mathrm{M} \mathrm{KOH}$ for the $\mathrm{La}_{1-\mathrm{x}} \mathrm{Sr}_{\mathrm{x}} \mathrm{CoO}_{3}$ series, (b) measured for $\mathrm{La}_{0.5} \mathrm{Sr}_{0.5} \mathrm{CoO}_{3-\delta}$ (LSC05) 0.1, 0.3 and $1 \mathrm{M} \mathrm{KOH}$ and $\mathrm{LiOH}$ and (c) measured for $\mathrm{La}_{0.5} \mathrm{Sr}_{0.5} \mathrm{CoO}_{3-\delta}(\mathrm{LSC05})$ in $1 \mathrm{M} \mathrm{LiOH}, \mathrm{NaOH}, \mathrm{KOH}$ and $\mathrm{CsOH}$.

To assess if such behavior is ubiquitous to every Co-based catalysts, we then investigated the behavior of electrodeposited amorphous cobalt oxyhydroxide films in different electrolytes. First, we should recall that the electrocatalytic activity of such amorphous catalysts is greatly affected by the presence of Fe-impurities in the alkaline electrolytes, ${ }^{12,20,21,23,49-53}$ and that the origin for such effects is still under intensive debate. For Fe-free electrolytes and films, the OER activity for $\mathrm{CoOOH}$ is found to be dependent on the concentration of $\mathrm{KOH}$ (Figure 4a). Alike for Co-based perovskites, this enhanced OER activity is explained by an increase of the preexponential factor rather than by a decrease of the apparent activation energy (Figure 4b). Nevertheless, unlike for perovskites, this concentration effect depends on the nature of the alkalication. Hence, no concentration dependence was observed with $\mathrm{LiOH}$ (Figure 4a) for which even though the pre-exponential factor is found to increase with the concentration of $\mathrm{LiOH}$, this effect is counter-balanced by the increase of the activation energy (Figure 4b) following the previously discussed compensation effect. Overall, the OER activity for amorphous $\mathrm{CoOOH}$ films is sensitive to the nature of the alkali-cation with $\mathrm{Cs}^{+}>\mathrm{Na}^{+} \approx \mathrm{K}^{+}>\mathrm{Li}^{+}$(Figure $4 \mathrm{c}$ ), alike what was recently reported for Fe-free $\mathrm{NiOOH}$ films by Koper and coworkers. ${ }^{48}$ Additionally, this dependence is only mild for $\mathrm{K}^{+}, \mathrm{Na}^{+}$and $\mathrm{Cs}^{+}$with only a factor of 2-3 in enhancement for the OER activity, while $\mathrm{Li}^{+}$shows an order of magnitude in decrease when compared to $\mathrm{K}^{+}$, similarly to our previous observations. ${ }^{24}$ Furthermore, a decrease of the activation energy could explain this increase in OER activity (Figure 4d). Nevertheless, the trend is not as conclusive as for Fe-containing $\mathrm{CoOOH}$ films, as discussed below. 

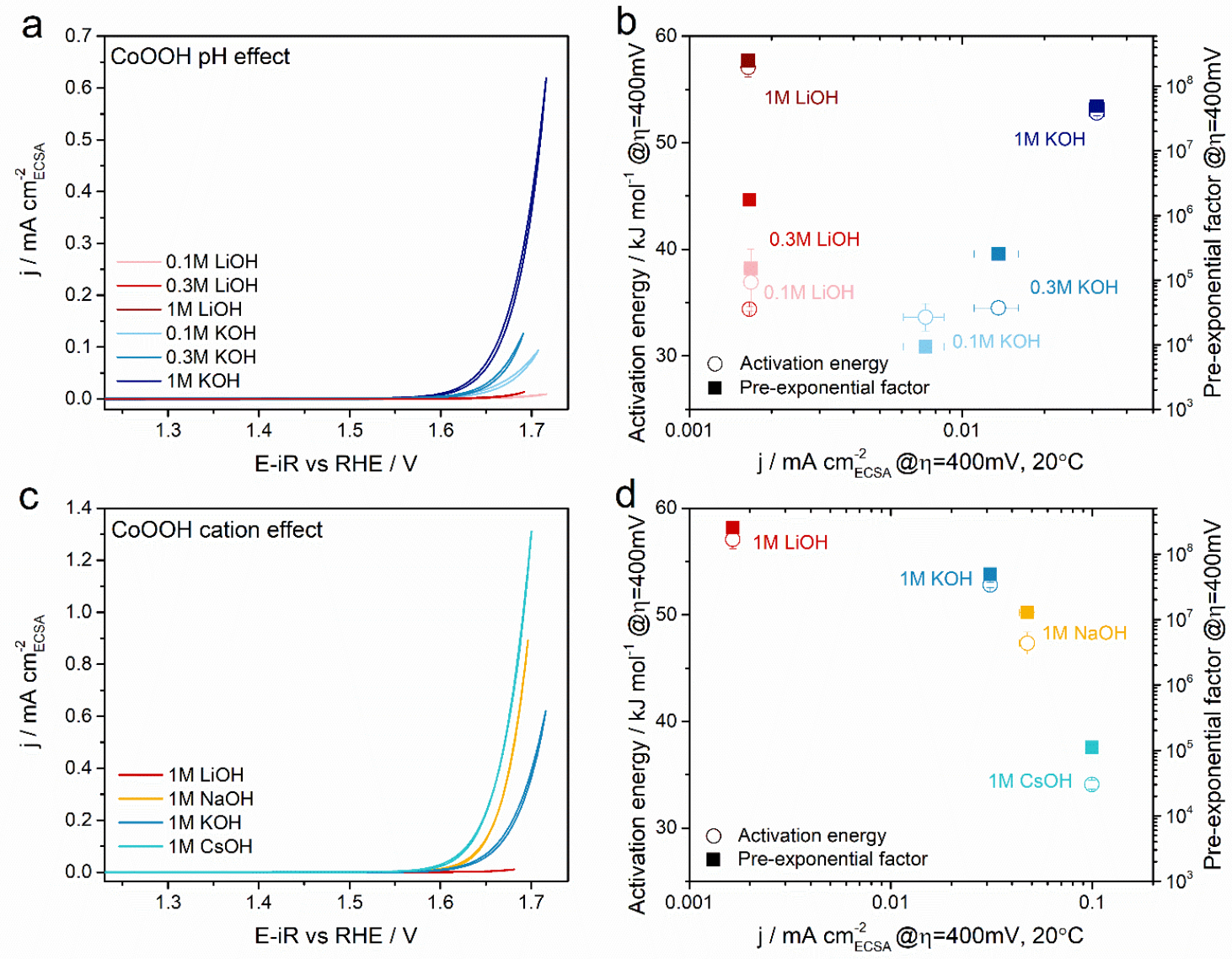

Figure 4. (a) $\mathrm{CV}$ curves for $\mathrm{CoOOH}$ measured at $20^{\circ} \mathrm{C}$ and (b) corresponding apparent activation energies and pre-exponential factors as a function of the $\mathrm{OER}$ activity at $20^{\circ} \mathrm{C}$ and $\eta=$ $400 \mathrm{mV}$ in $0.1,0.3$ and $1 \mathrm{M} \mathrm{LiOH}$ and $\mathrm{KOH}$. (c) $\mathrm{CV}$ curves for $\mathrm{CoOOH}$ measured at $20^{\circ} \mathrm{C}$ and (b) corresponding apparent activation energies and pre-exponential factors as a function of the OER activity at $20^{\circ} \mathrm{C}$ and $\eta=400 \mathrm{mV}$ in $1 \mathrm{M} \mathrm{LiOH}, \mathrm{NaOH}, \mathrm{KOH}$ and $\mathrm{CsOH}$.

Unlike for Fe-free $\mathrm{CoOOH}$, the OER activity for $\mathrm{Co}_{0.5} \mathrm{Fe}_{0.5} \mathrm{OOH}$ films shows no dependence on the concentration of alkali salts with both $\mathrm{LiOH}$ and $\mathrm{KOH}$ (Figure 5a). This observation is in line with our previous measurements for Ni-oxyhydroxide Fe-containing films for which almost no concentration dependence was observed. ${ }^{24}$ Once again, this concentration-independent OER kinetics is found to be accompanied with an almost perfect compensation of the increase of the 
activation energy by an increase of the pre-exponential factor (Figure 5b). More interestingly, the OER kinetics for the $\mathrm{Co}_{0.5} \mathrm{Fe}_{0.5} \mathrm{OOH}$ films are found to be greatly dependent on the alkali-cation with $\mathrm{Cs}^{+}>\mathrm{K}^{+}>\mathrm{Na}^{+}>\mathrm{Li}^{+}$(Figure 5c and 5d) following the decrease of the activation energy from $\approx 60 \mathrm{~kJ} / \mathrm{mol}( \pm 1)$ in $1 \mathrm{M} \mathrm{LiOH}$ to $\approx 20 \mathrm{~kJ} / \mathrm{mol}( \pm 1)$ in $1 \mathrm{M} \mathrm{CsOH}$ (Figure $5 \mathrm{~d})$.
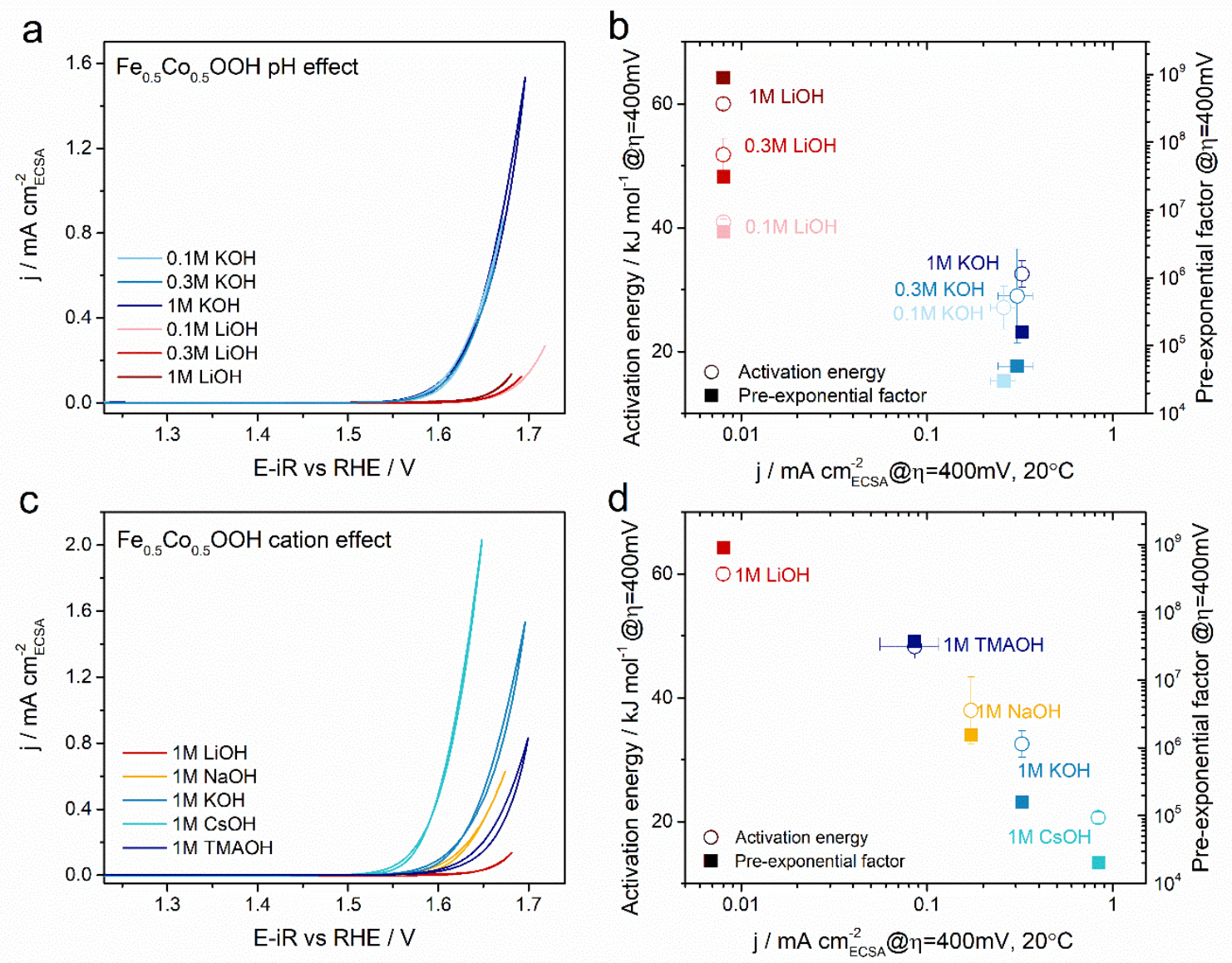

Figure 5. (a) $\mathrm{CV}$ curves for $\mathrm{Fe}_{0.5} \mathrm{Co}_{0.5} \mathrm{OOH}$ measured at $20^{\circ} \mathrm{C}$ and (b) corresponding apparent activation energies and pre-exponential factors as a function of the OER activity at $20^{\circ} \mathrm{C}$ and $\eta=$ $400 \mathrm{mV}$ in $0.1,0.3$ and $1 \mathrm{M} \mathrm{LiOH}$ and $\mathrm{KOH}$. (c) $\mathrm{CV}$ curves for $\mathrm{Fe}_{0.5} \mathrm{Co}_{0.5} \mathrm{OOH}$ measured at $20^{\circ} \mathrm{C}$ and (b) corresponding apparent activation energies and pre-exponential factors as a function of the OER activity at $20^{\circ} \mathrm{C}$ and $\eta=400 \mathrm{mV}$ in $1 \mathrm{M} \mathrm{LiOH}, \mathrm{NaOH}, \mathrm{KOH}$ and $\mathrm{CsOH}$. 
Having demonstrated the effect of alkali-cation for Fe-containing films, one can then try to rationalize these observations. Indeed, our results suggest that the physical origin for both the concentration dependence observed for Fe-free films (as well as for perovskites) and the cation dependence measured for Fe-containing films (and to a lesser extend Fe-free films) is different. For Fe-free films, the concentration dependence in $\mathrm{KOH}$ arises from an increase of the apparent pre-exponential factor (Figure 4b). This increase can be explained by a greater concentration of active sites $(* \mathrm{O})$ following a partial deprotonation of the hydroxides sites at greater hydroxyls activity (i.e. greater concentration). Additionally, this increase can also arise from a change in the entropy of activation $\Delta \mathrm{S}^{*}$ reflecting a modification of the electrolyte structure surrounding the active sites and a perturbation of the ions reorganization at the water/catalyst interface. In fact, as previously discussed for the HER, when transferring protons across the electrochemical interface from the inner to the outer Helmotz plane, protons undergo a change in configurational entropy, ${ }^{33,44,54}$ this change being dependent on the strength of the electrical field. Therefore, strengthening the electrical field stiffens the water structure in the double layer ${ }^{55}$ and therefore increases the work necessary to transfer protons or ions through the interface. Rather, for Fecontaining films, the apparent activation energies are modified by changing the nature of the alkali-cation (Figure 5d), which suggests a strong interaction of the whole film thickness with the electrolyte. This modification can be ascribed to the so-called self-insertion of cations in the film upon operation which modifies the chemical composition and the physical properties of the films and therefore the binding energies of intermediates. In order to better understand the major factor influencing this change in intermediates binding energies, the OER kinetics previously measured for $\mathrm{Co}_{0.5} \mathrm{Fe}_{0.5} \mathrm{OOH}$ with $\mathrm{MOH}$ solutions, $\mathrm{M}$ being an alkali-cation, were compared with the one measured for a large hydrophobic cation, namely tetramethylammonium $\mathrm{TMA}^{+}$(Figure $5 \mathrm{c}$ and 
5d). Doing so, we found once again that the subsequent modification of the OER kinetics when using $\mathrm{TMA}^{+}$is related to a change in the apparent activation energy (Figure 5d), therefore showing that such self-insertion is certainly not limited to alkali cations.

\section{CONCLUSIONS}

In conclusion, through a systematic temperature dependence study we could establish the physical origin for the concentration- and cation-dependence of the OER kinetics for a series of Co-based catalysts. First, we discussed how challenging the obtention of reliable experimental kinetic parameters for the OER catalysts is. Especially, we emphasize that the proper determination of exchange current densities for OER catalysts from a simple Tafel analysis may not be an adequate approach. Indeed, the modification of the surface coverage ${ }^{32}$ or of the catalyst nature $^{41}$ with the applied potential result in Tafel slopes that do not match simple microkinetic models, thus preventing the extraction of meaningful exchange current densities. In this work, we overcame this issue by comparing the kinetics of different materials at a given overpotential. Applying this methodology, we confirmed that when modifying the physical properties of perovskites-based OER catalysts through a substitution strategy, the apparent activation energies are modified, reflecting a modification of the enthalpy of formation for the intermediates of the reaction. Furthermore, we could demonstrate for the first time for the OER that similarly to other

fields such as biology, the experimentally determined apparent pre-exponential factors linearly trend with the apparent activation energies, following a so-called compensation effect. Having established the validity of the methodology, we then turned our attention to the study of the effect of the electrolyte composition on amorphous cobalt oxyhydroxides films. Doing so, we confirmed that Fe incorporation into these amorphous films greatly affects their OER activity. 
Comparing the apparent activation energies and the pre-exponential factors extracted from the temperature dependence study, we demonstrate that the concentration dependence observed for Fe-free films (as well as for the $\mathrm{La}_{1-\mathrm{x}} \mathrm{Sr}_{\mathrm{x}} \mathrm{CoO}_{3-\delta}$ series) originates from an increase in the preexponential factor as a result of either an increase of active sites or a change in the entropy of activation. These effects, which can hardly be capture by either operando measurements or by classical computational methods, are nicely captured by our experimental methodology which reinforces its significance to the field. Instead, for Fe-containing films, no concentration dependence was found but a large cation dependence is observed. This cation dependence arises from a change in the apparent activation energies, which reflect a change in the enthalpy of formation of the intermediates, certainly as a result of cation self-insertion into the film.

Eventually, more remains to be done to fully uncover the concentration and cation dependence of the OER activity for these catalysts, especially from a theoretical point of view. However, we believe that such findings open up new avenues to intrinsically tune the electrocatalytic activity of OER catalysts by controlling the water/catalysts interactions in the vicinity of the active sites. Finally, we believe that this experimental methodology can be applied to understand complex electrolyte effects previously reported for other complex reactions such as the ORR or $\mathrm{CO}_{2}$ reduction.

\section{ASSOCIATED CONTENT}

\section{Supporting Information}

The Supporting Information is available free of charge on the ACS Publications website at DOI: Additional experimental details and characterization data (PDF) 


\section{AUTHOR INFORMATION}

\section{Corresponding Author}

*E-mail: alexis.grimaud@ college-de-france.fr. Tel: +33 144271189.

\section{Notes}

The authors declare no competing financial interest.

\section{ACKNOWLEDGMENTS}

A.G. acknowledges financial support from the ANR MIDWAY (Project No. ANR-17-CE050008). Y.D. acknowledge funding from NTU-PSL program.

\section{REFERENCES}

(1) Hong, W. T.; Risch, M.; Stoerzinger, K. A.; Grimaud, A.; Suntivich, J.; Shao-Horn, Y. Toward the Rational Design of Non-Precious Transition Metal Oxides for Oxygen Electrocatalysis. Energy Environ. Sci. 2015, 8 (5), 1404-1427. https://doi.org/10.1039/C4EE03869J.

(2) Fabbri, E.; Habereder, A.; Waltar, K.; Kötz, R.; Schmidt, T. J. Developments and Perspectives of Oxide-Based Catalysts for the Oxygen Evolution Reaction. Catal. Sci. Technol. 2014, 4 (11), 38003821. https://doi.org/10.1039/C4CY00669K.

(3) Shen, P. K.; Wang, C.-Y.; Jiang, S. P.; Sun, X.; Zhang, J. Electrochemical Energy. 619.

(4) Lee, J.; Kim, I.; Park, S. Boosting Stability and Activity of Oxygen Evolution Catalyst in Acidic Medium: Bimetallic Ir-Fe Oxides on Reduced Graphene Oxide Prepared through Ultrasonic Spray Pyrolysis. ChemCatChem 2019, 11 (11), 2615-2623. https://doi.org/10.1002/cctc.201900287.

(5) Hong, W. T.; Welsch, R. E.; Shao-Horn, Y. Descriptors of Oxygen-Evolution Activity for Oxides: A Statistical Evaluation. J. Phys. Chem. C 2016, 120 (1), 78-86. https://doi.org/10.1021/acs.jpcc.5b10071.

(6) Mefford, J. T.; Rong, X.; Abakumov, A. M.; Hardin, W. G.; Dai, S.; Kolpak, A. M.; Johnston, K. P.; Stevenson, K. J. Water Electrolysis on La 1- x Sr x CoO 3- $\delta$ Perovskite Electrocatalysts. Nature communications 2016, 7, 11053.

(7) Duan, Y.; Sun, S.; Sun, Y.; Xi, S.; Chi, X.; Zhang, Q.; Ren, X.; Wang, J.; Ong, S. J. H.; Du, Y. Mastering Surface Reconstruction of Metastable Spinel Oxides for Better Water Oxidation. Advanced Materials 2019, 31 (12), 1807898.

(8) Huang, Z.-F.; Song, J.; Du, Y.; Xi, S.; Dou, S.; Nsanzimana, J. M. V.; Wang, C.; Xu, Z. J.; Wang, X. Chemical and Structural Origin of Lattice Oxygen Oxidation in Co-Zn Oxyhydroxide Oxygen Evolution Electrocatalysts. Nat Energy 2019, 4 (4), 329-338. https://doi.org/10.1038/s41560-0190355-9.

(9) Zhou, Y.; Sun, S.; Song, J.; Xi, S.; Chen, B.; Du, Y.; Fisher, A. C.; Cheng, F.; Wang, X.; Zhang, H.; et al. Enlarged Co: $\mathrm{O}$ Covalency in Octahedral Sites Leading to Highly Efficient Spinel Oxides for Oxygen 
Evolution Reaction. Adv. Mater. 2018, 30 (32), 1802912.

https://doi.org/10.1002/adma.201802912.

(10) Wu, T.; Sun, S.; Song, J.; Xi, S.; Du, Y.; Chen, B.; Sasangka, W. A.; Liao, H.; Gan, C. L.; Scherer, G. G.; et al. Iron-Facilitated Dynamic Active-Site Generation on Spinel CoAl2O4 with Self-Termination of Surface Reconstruction for Water Oxidation. Nat Catal 2019. https://doi.org/10.1038/s41929019-0325-4.

(11) Yang, C.; Batuk, M.; Jacquet, Q.; Rousse, G.; Yin, W.; Zhang, L.; Hadermann, J.; Abakumov, A. M.; Cibin, G.; Chadwick, A. Revealing PH-Dependent Activities and Surface Instabilities for Ni-Based Electrocatalysts during the Oxygen Evolution Reaction. ACS Energy Letters 2018, 3 (12), 28842890.

(12) Trotochaud, L.; Young, S. L.; Ranney, J. K.; Boettcher, S. W. Nickel-Iron Oxyhydroxide OxygenEvolution Electrocatalysts: The Role of Intentional and Incidental Iron Incorporation. J. Am. Chem. Soc. 2014, 136 (18), 6744-6753. https://doi.org/10.1021/ja502379c.

(13) Abidat, I.; Bouchenafa-Saib, N.; Habrioux, A.; Comminges, C.; Canaff, C.; Rousseau, J.; Napporn, T. W.; Dambournet, D.; Borkiewicz, O.; Kokoh, K. B. Electrochemically Induced Surface Modifications of Mesoporous Spinels $\left(\mathrm{CO}_{3} \mathrm{O}_{4-\delta}, \mathrm{MnCo}_{2} \mathrm{O}_{4-\delta}, \mathrm{NiCo}_{2} \mathrm{O}_{4-\delta}\right.$ ) as the Origin of the OER Activity and Stability in Alkaline Medium. J. Mater. Chem. A 2015, 3 (33), 17433-17444. https://doi.org/10.1039/C5TA04437E.

(14) Man, I. C.; Su, H.-Y.; Calle-Vallejo, F.; Hansen, H. A.; Martínez, J. I.; Inoglu, N. G.; Kitchin, J.; Jaramillo, T. F.; Nørskov, J. K.; Rossmeisl, J. Universality in Oxygen Evolution Electrocatalysis on Oxide Surfaces. ChemCatChem 2011, 3 (7), 1159-1165.

(15) Grimaud, A.; Diaz-Morales, O.; Han, B.; Hong, W. T.; Lee, Y.-L.; Giordano, L.; Stoerzinger, K. A.; Koper, M. T. M.; Shao-Horn, Y. Activating Lattice Oxygen Redox Reactions in Metal Oxides to Catalyse Oxygen Evolution. Nature Chem 2017, 9 (5), 457-465. https://doi.org/10.1038/nchem.2695.

(16) Yang, C.; Laberty-Robert, C.; Batuk, D.; Cibin, G.; Chadwick, A. V.; Pimenta, V.; Yin, W.; Zhang, L.; Tarascon, J.-M.; Grimaud, A. Phosphate Ion Functionalization of Perovskite Surfaces for Enhanced Oxygen Evolution Reaction. The journal of physical chemistry letters 2017, 8 (15), 3466-3472.

(17) Burke, M. S.; Kast, M. G.; Trotochaud, L.; Smith, A. M.; Boettcher, S. W. Cobalt-Iron (Oxy)Hydroxide Oxygen Evolution Electrocatalysts: The Role of Structure and Composition on Activity, Stability, and Mechanism. J. Am. Chem. Soc. 2015, 137 (10), 3638-3648. https://doi.org/10.1021/jacs.5b00281.

(18) Burke, M. S.; Enman, L. J.; Batchellor, A. S.; Zou, S.; Boettcher, S. W. Oxygen Evolution Reaction Electrocatalysis on Transition Metal Oxides and (Oxy)Hydroxides: Activity Trends and Design Principles. Chem. Mater. 2015, 27 (22), 7549-7558. https://doi.org/10.1021/acs.chemmater.5b03148.

(19) Enman, L. J.; Stevens, M. B.; Dahan, M. H.; Nellist, M. R.; Toroker, M. C.; Boettcher, S. W. Operando X-Ray Absorption Spectroscopy Shows Iron Oxidation Is Concurrent with Oxygen Evolution in Cobalt-Iron (Oxy)Hydroxide Electrocatalysts. Angew. Chem. Int. Ed. 2018, 57 (39), 12840-12844. https://doi.org/10.1002/anie.201808818.

(20) Zhang, T.; Nellist, M. R.; Enman, L. J.; Xiang, J.; Boettcher, S. W. Modes of Fe Incorporation in CoFe (Oxy)Hydroxide Oxygen Evolution Electrocatalysts. ChemSusChem 2019, 12 (9), 2015-2021. https://doi.org/10.1002/cssc.201801975.

(21) Michael, J. D.; Demeter, E. L.; Illes, S. M.; Fan, Q.; Boes, J. R.; Kitchin, J. R. Alkaline Electrolyte and Fe Impurity Effects on the Performance and Active-Phase Structure of NiOOH Thin Films for OER Catalysis Applications. The Journal of Physical Chemistry C 2015, 119 (21), 11475-11481. 
(22) Zaffran, J.; Stevens, M. B.; Trang, C. D. M.; Nagli, M.; Shehadeh, M.; Boettcher, S. W.; Caspary Toroker, M. Influence of Electrolyte Cations on $\mathrm{Ni}(\mathrm{Fe}) \mathrm{OOH}$ Catalyzed Oxygen Evolution Reaction. Chem. Mater. 2017, 29 (11), 4761-4767. https://doi.org/10.1021/acs.chemmater.7b00517.

(23) Trześniewski, B. J.; Diaz-Morales, O.; Vermaas, D. A.; Longo, A.; Bras, W.; Koper, M. T. M.; Smith, W. A. In Situ Observation of Active Oxygen Species in Fe-Containing Ni-Based Oxygen Evolution Catalysts: The Effect of PH on Electrochemical Activity. J. Am. Chem. Soc. 2015, 137 (48), 1511215121. https://doi.org/10.1021/jacs.5b06814.

(24) Yang, C.; Fontaine, O.; Tarascon, J.-M.; Grimaud, A. Chemical Recognition of Active Oxygen Species on the Surface of Oxygen Evolution Reaction Electrocatalysts. Angewandte Chemie 2017, 129 (30), 8778-8782.

(25) Merrill, M.; Worsley, M.; Wittstock, A.; Biener, J.; Stadermann, M. Determination of the "NiOOH" Charge and Discharge Mechanisms at Ideal Activity. Journal of Electroanalytical Chemistry 2014, 717-718, 177-188. https://doi.org/10.1016/j.jelechem.2014.01.022.

(26) Ali-Löytty, H.; Louie, M. W.; Singh, M. R.; Li, L.; Sanchez Casalongue, H. G.; Ogasawara, H.; Crumlin, E. J.; Liu, Z.; Bell, A. T.; Nilsson, A.; et al. Ambient-Pressure XPS Study of a Ni-Fe Electrocatalyst for the Oxygen Evolution Reaction. J. Phys. Chem. C 2016, 120 (4), 2247-2253. https://doi.org/10.1021/acs.jpcc.5b10931.

(27) Zhu, K.; Zhu, X.; Yang, W. Application of In Situ Techniques for the Characterization of NiFe-Based Oxygen Evolution Reaction (OER) Electrocatalysts. Angewandte Chemie International Edition 2019, 58 (5), 1252-1265. https://doi.org/10.1002/anie.201802923.

(28) Yeo, B. S.; Bell, A. T. In Situ Raman Study of Nickel Oxide and Gold-Supported Nickel Oxide Catalysts for the Electrochemical Evolution of Oxygen. J. Phys. Chem. C 2012, 116 (15), 83948400. https://doi.org/10.1021/jp3007415.

(29) Tkalych, A. J.; Zhuang, H. L.; Carter, E. A. A Density Functional + U Assessment of Oxygen Evolution Reaction Mechanisms on $\beta-\mathrm{NiOOH}$. ACS Catal. 2017, 7 (8), 5329-5339. https://doi.org/10.1021/acscatal.7b00999.

(30) Huang, B.; Muy, S.; Feng, S.; Katayama, Y.; Lu, Y.-C.; Chen, G.; Shao-Horn, Y. Non-Covalent Interactions in Electrochemical Reactions and Implications in Clean Energy Applications. Physical Chemistry Chemical Physics 2018, 20 (23), 15680-15686.

(31) Gileadi, E. Physical Electrochemistry; Wiley-VCH Verlag GmbH \& Co. KGaA, 2011.

(32) Shinagawa, T.; Garcia-Esparza, A. T.; Takanabe, K. Insight on Tafel Slopes from a Microkinetic Analysis of Aqueous Electrocatalysis for Energy Conversion. Sci Rep 2015, 5 (1), 13801. https://doi.org/10.1038/srep13801.

(33) Zeradjanin, A. R.; Vimalanandan, A.; Polymeros, G.; Topalov, A. A.; Mayrhofer, K. J.; Rohwerder, M. Balanced Work Function as a Driver for Facile Hydrogen Evolution Reaction-Comprehension and Experimental Assessment of Interfacial Catalytic Descriptor. Physical Chemistry Chemical Physics 2017, 19 (26), 17019-17027.

(34) Koper, M. T. M. Thermodynamic Theory of Multi-Electron Transfer Reactions: Implications for Electrocatalysis. Journal of Electroanalytical Chemistry 2011, 660 (2), 254-260. https://doi.org/10.1016/j.jelechem.2010.10.004.

(35) Lide, D. R.; Baysinger, G.; Chemistry, S.; Berger, L. I.; Goldberg, R. N.; Kehiaian, H. V. CRC Handbook of Chemistry and Physics. 2661.

(36) Bard, A. J.; Faulkner, L. R. Electrochemical Methods: Fundamentals and Applications, 2nd ed.; Wiley, 2000.

(37) Grimaud, A.; Diaz-Morales, O.; Han, B.; Hong, W. T.; Lee, Y.-L.; Giordano, L.; Stoerzinger, K. A.; Koper, M. T.; Shao-Horn, Y. Activating Lattice Oxygen Redox Reactions in Metal Oxides to Catalyse Oxygen Evolution. Nature chemistry 2017, 9 (5), 457. 
(38) Grimaud, A.; May, K. J.; Carlton, C. E.; Lee, Y.-L.; Risch, M.; Hong, W. T.; Zhou, J.; Shao-Horn, Y. Double Perovskites as a Family of Highly Active Catalysts for Oxygen Evolution in Alkaline Solution. Nature communications 2013, 4, 2439.

(39) Conway, B. E.; Bockris, J. O.; Linton, H. Proton Conductance and the Existence of the $\mathrm{H}_{3} \mathrm{O}$ Ion. The Journal of Chemical Physics 1956, 24 (4), 834-850. https://doi.org/10.1063/1.1742619.

(40) Matsumoto, Y.; Manabe, H.; Sato, E. Oxygen Evolution on Lal_Sr CoO Electrodes in Alkaline Solutions. 4.

(41) Mefford, J. T.; Zhao, Z.; Bajdich, M.; Chueh, W. C. Interpreting Tafel Behavior of Consecutive Electrochemical Reactions through Combined Thermodynamic and Steady State Microkinetic Approaches. Energy Environ. Sci. 2020. https://doi.org/10.1039/C9EE02697E.

(42) Bockris, J. O.; Otagawa, T. Mechanism of Oxygen Evolution on Perovskites. J. Phys. Chem. 1983, 87 (15), 2960-2971. https://doi.org/10.1021/j100238a048.

(43) Zhang, R.; Pearce, P. E.; Duan, Y.; Dubouis, N.; Marchandier, T.; Grimaud, A. The Importance of Water Structure and Catalyst-Electrolyte Interface on the Design of Water Splitting Catalysts. Chem. Mater. 2019, acs.chemmater.9b02318. https://doi.org/10.1021/acs.chemmater.9b02318.

(44) Shinagawa, T.; Takanabe, K. New Insight into the Hydrogen Evolution Reaction under Buffered Near-Neutral PH Conditions: Enthalpy and Entropy of Activation. J. Phys. Chem. C 2016, 120 (42), 24187-24196. https://doi.org/10.1021/acs.jpcc.6b07954.

(45) Conner, Wm. C. A General Explanation for the Compensation Effect: The Relationship between $\triangle \mathrm{S} \ddagger$ and Activation Energy. Journal of Catalysis 1982, 78 (1), 238-246. https://doi.org/10.1016/0021-9517(82)90303-7.

(46) Yelon, A.; Movaghar, B.; Crandall, R. S. Multi-Excitation Entropy: Its Role in Thermodynamics and Kinetics. Rep. Prog. Phys. 2006, 69 (4), 1145-1194. https://doi.org/10.1088/0034-4885/69/4/R04.

(47) Anderson, A. B.; Roques, J.; Mukerjee, S.; Murthi, V. S.; Markovic, N. M.; Stamenkovic, V. Activation Energies for Oxygen Reduction on Platinum Alloys: Theory and Experiment. The Journal of Physical Chemistry B 2005, 109 (3), 1198-1203.

(48) Garcia, A. C.; Touzalin, T.; Nieuwland, C.; Perini, N.; Koper, M. T. M. Enhancement of Oxygen Evolution Activity of NiOOH by Electrolyte Alkali Cations. Angew. Chem. Int. Ed. 2019. https://doi.org/10.1002/anie.201905501.

(49) Gong, L.; Ren, D.; Deng, Y.; Yeo, B. S. Efficient and Stable Evolution of Oxygen Using PulseElectrodeposited Ir/Ni Oxide Catalyst in Fe-Spiked KOH Electrolyte. ACS Appl. Mater. Interfaces 2016, 8 (25), 15985-15990. https://doi.org/10.1021/acsami.6b01888.

(50) Gong, L.; Chng, X. Y. E.; Du, Y.; Xi, S.; Yeo, B. S. Enhanced Catalysis of the Electrochemical Oxygen Evolution Reaction by Iron(III) Ions Adsorbed on Amorphous Cobalt Oxide. ACS Catal. 2018, 8 (2), 807-814. https://doi.org/10.1021/acscatal.7b03509.

(51) Klaus, S.; Trotochaud, L.; Cheng, M.-J.; Head-Gordon, M.; Bell, A. T. Experimental and Computational Evidence of Highly Active Fe Impurity Sites on the Surface of Oxidized Au for the Electrocatalytic Oxidation of Water in Basic Media. ChemElectroChem 2016, 3 (1), 66-73. https://doi.org/10.1002/celc.201500364.

(52) Li, N.; Bediako, D. K.; Hadt, R. G.; Hayes, D.; Kempa, T. J.; von Cube, F.; Bell, D. C.; Chen, L. X.; Nocera, D. G. Influence of Iron Doping on Tetravalent Nickel Content in Catalytic Oxygen Evolving Films. Proc Natl Acad Sci USA 2017, 114 (7), 1486-1491. https://doi.org/10.1073/pnas.1620787114.

(53) Corrigan, D. A. The Catalysis of the Oxygen Evolution Reaction by Iron Impurities in Thin Film Nickel Oxide Electrodes. J. Electrochem. Soc. 1987, 134 (2), 377. https://doi.org/10.1149/1.2100463. 
(54) Rossmeisl, J.; Chan, K.; Skúlason, E.; Björketun, M. E.; Tripkovic, V. On the PH Dependence of Electrochemical Proton Transfer Barriers. Catalysis Today 2016, 262, 36-40. https://doi.org/10.1016/j.cattod.2015.08.016.

(55) Ledezma-Yanez, I.; Wallace, W. D. Z.; Sebastián-Pascual, P.; Climent, V.; Feliu, J. M.; Koper, M. T. M. Interfacial Water Reorganization as a PH-Dependent Descriptor of the Hydrogen Evolution Rate on Platinum Electrodes. Nat Energy 2017, 2 (4), 17031.

https://doi.org/10.1038/nenergy.2017.31. 